\title{
Editorial
}

\section{Harmful Chemicals in the Environment 2016}

\author{
Jian Lu, ${ }^{1}$ Patrick C. Wilson, ${ }^{2}$ Xianghua Wen, ${ }^{3}$ Qiang Jin, ${ }^{4}$ and Jun $\mathrm{Wu}^{5}$ \\ ${ }^{1}$ Key Laboratory of Coastal Environmental Processes and Ecological Remediation, Yantai Institute of Coastal Zone Research, \\ Chinese Academy of Sciences, Yantai, Shandong 264003, China \\ ${ }^{2}$ Soil and Water Science Department, University of Florida, Gainesville, FL 32611, USA \\ ${ }^{3}$ School of Environment, Tsinghua University, Beijing 100084, China \\ ${ }^{4}$ School of Environmental Science and Engineering, Shanghai Jiao Tong University, Shanghai 200240, China \\ ${ }^{5}$ Department of Civil and Architectural Engineering, University of Wyoming, Laramie, WY 82071, USA
}

Correspondence should be addressed to Jian Lu; jlu@yic.ac.cn

Received 4 August 2016; Accepted 4 August 2016

Copyright (C) 2016 Jian Lu et al. This is an open access article distributed under the Creative Commons Attribution License, which permits unrestricted use, distribution, and reproduction in any medium, provided the original work is properly cited.

Chemicals, especially synthetic chemicals, might post potential risks to ecosystem and human health if they excessively exist in the environment due to overuse, accidental spill, accumulation, and so forth. Therefore, it is urgent and important to explore the chemicals in the environment. This special issue is aimed at providing useful information on analytical methods for monitoring harmful chemicals in various environments, pollution control processes and technologies for harmful chemicals, the fate of harmful chemicals in natural and engineered environments, and ecological risks posed by the harmful chemicals. Therefore, some submitted articles are selected to show the current research frontiers in measurement, fate, ecological risks, and remediation of harmful chemicals in the environment.

Nutrient control was extensively explored. Therefore, the influences of different controlled irrigation and drainage conditions on dynamics of nitrogen $(\mathrm{N})$ and phosphorus $(\mathrm{P})$ were studied by S. Gao et al. and J. Cui et al. Controlled irrigation and drainage seemed to be an efficient method for controlling $\mathrm{N}$ and $\mathrm{P}$.

Removal of heavy metal in water was tried using different methods. Y. Guo et al. reported a novel approach for removing aqueous $\mathrm{Cu}(\mathrm{II})$. They adopted wheat straw with alkali pretreatment to adsorb $\mathrm{Cu}$ (II) under acidic condition and optimized alkali pretreatment conditions. J. Liu et al. investigated adsorption properties of $\mathrm{Cr}(\mathrm{III})$ or $\mathrm{Cr}(\mathrm{VI})$ in the absence and presence of $\mathrm{Cu}(\mathrm{II})$ onto kaolin under $\mathrm{pH}$ of 2.0-7.0. They found that $\mathrm{pH}$ played a critical role in kaolin zeta potential.
Studies on organic pollution control were performed through different aspects. G. Zhu et al. used biological pretreatment reactors to remove organic pollutants from eutrophic water of Lake Taihu. Biological contact oxidation reactors-cascade biofilm reactor (CSBR) was proved to be efficient in low-molecular-weight organic pollution. X. Bian and J. Zhang explore photodegradation of sulfadiazine under ultraviolet (UV) light. The degradation of sulfadiazine in water followed the first-order kinetics with a half-life of $8.44 \mathrm{~min}$. Y. Dong et al. studied the promotion effect of methanoic acid on photoelectrocatalytic degradation of fulvic acid. They also discussed the degradation mechanism. S. Liu et al. focused on the natural degradation and ecotoxicity of nonivamide in seawater and tap water.

Ecological risks posed by pollutants were also paid attention to. J. Hussain et al. studied temporal distribution and ecological risk of polycyclic aromatic hydrocarbons in sediments of Changdang Lake, China.

$\mathrm{Z}$. Xu et al. brought us new knowledge on chemicals in the tailings dam. They paid attention to permeability characteristics of tailings during chemical-physical clogging process.

The articles of this special issue contributed to new information on monitoring, fate, risks, and treatment of harmful chemicals in the environment. We hope this special issue will have a long-term impact in the field of the environmental chemistry and technology. 


\section{Acknowledgments}

We would like to thank the Editorial Board for publishing our special issue. We thank the contributors for their valuable research articles submitted to our special issue. Special thanks and gratitude are extended to the reviewers for their critical and timely assessments of each paper.

Jian $\mathrm{Lu}$

Patrick C. Wilson

Xianghua Wen Qiang Jin

Jun $W u$ 

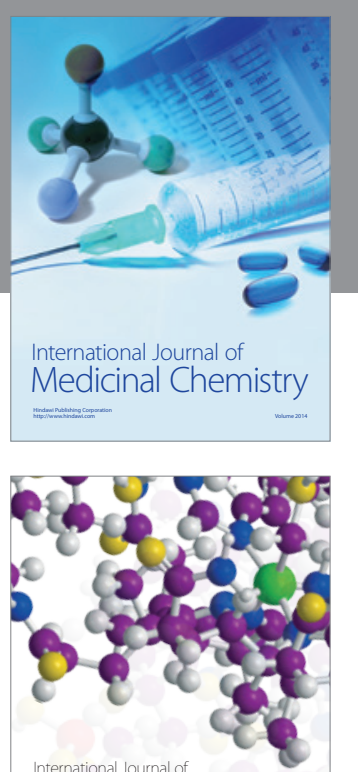

Carbohydrate Chemistry

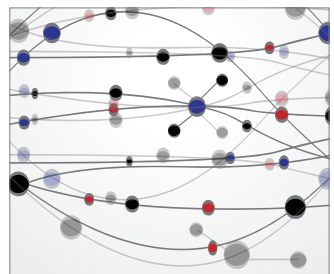

The Scientific World Journal
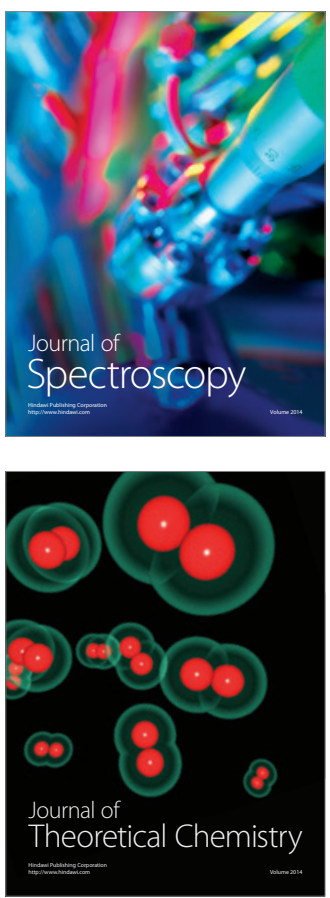
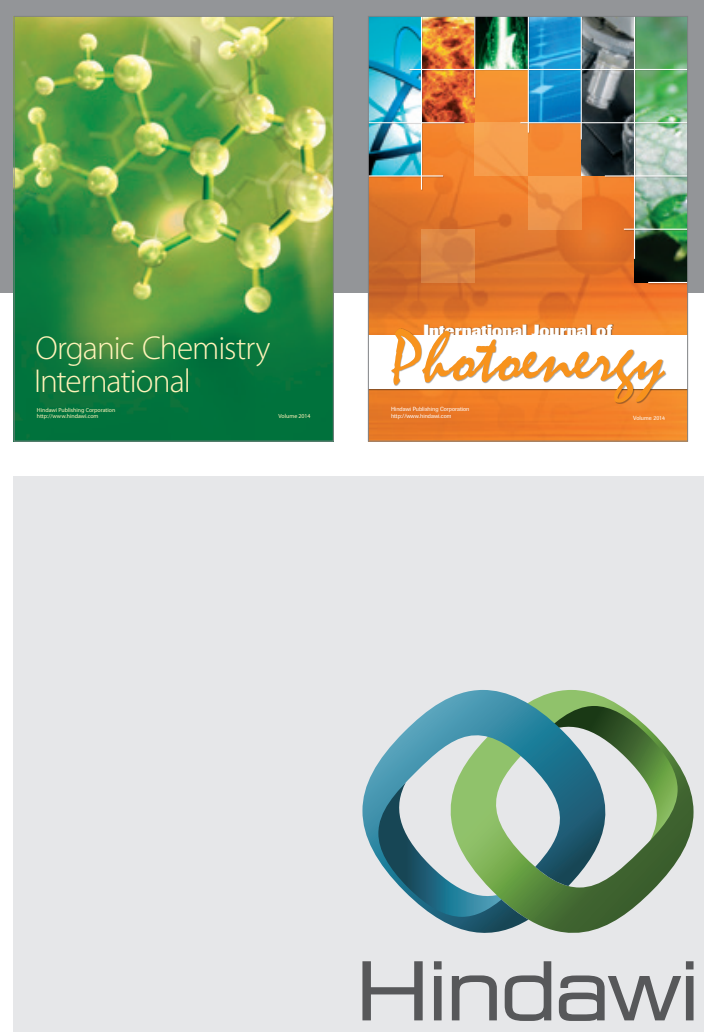

Submit your manuscripts at

http://www.hindawi.com

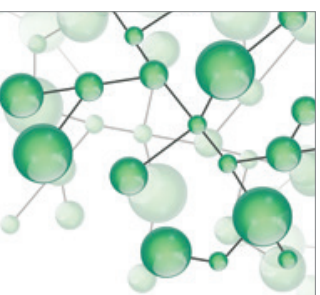

International Journal of

Inorganic Chemistry

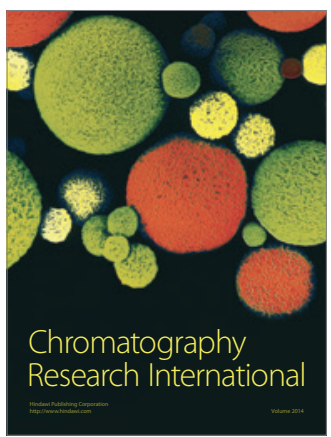

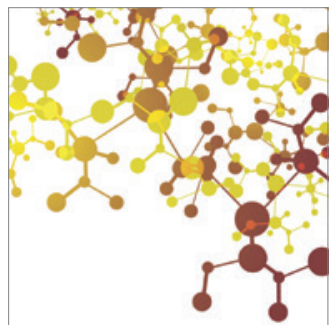

Applied Chemistry
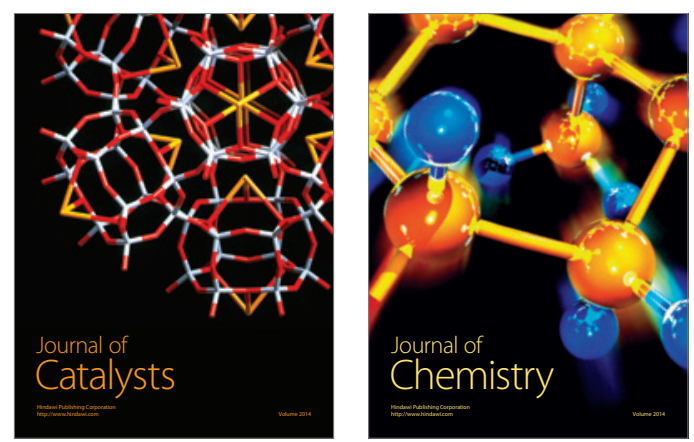
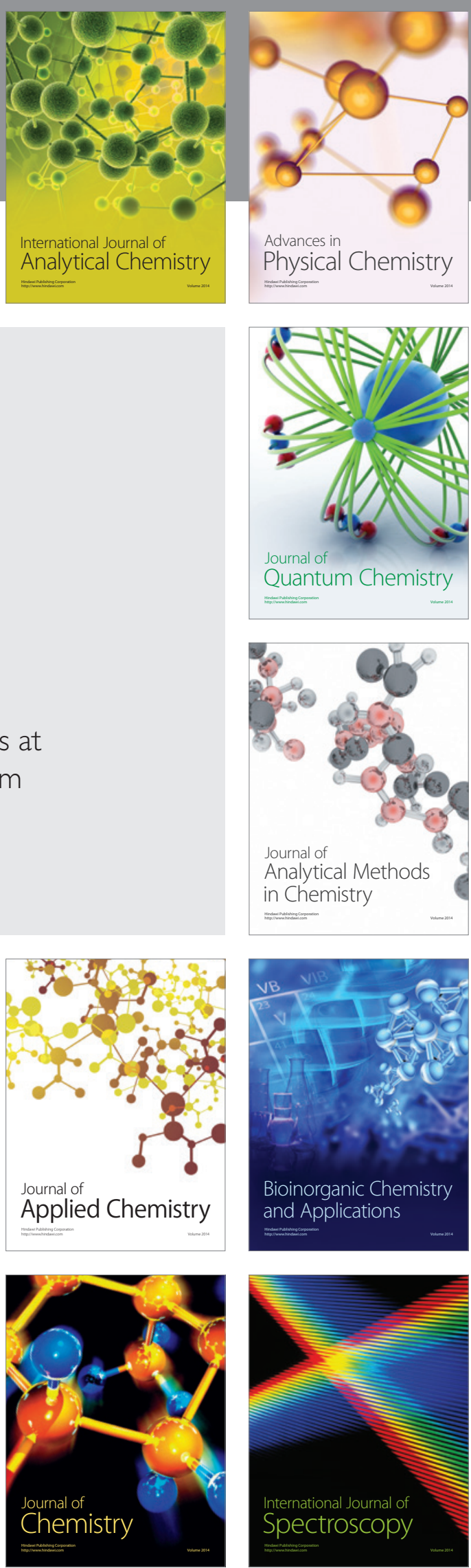